\title{
Supracervical hysterectomy - the vaginal route
}

\author{
Miłosz Wilczyński, Jarosław Cieślak, Andrzej Malinowski \\ Department of Surgical, Endoscopic and Oncologic Gynecology, Polish Mother's Memorial Hospital Research Institute, Lodz, Poland \\ Videosurgery Miniinv 2014; 9 (2): 207-212 \\ DOI: $10.5114 /$ wiitm.2014.41633
}

\begin{abstract}
Removal of the cervix during hysterectomy is not mandatory. There has been no irrefutable evidence so far that total hysterectomy is more beneficial to patients in terms of pelvic organ function. The procedure that leaves the cervix intact is called a subtotal hysterectomy. Traditional approaches to this surgery include laparoscopic and abdominal routes. Vaginal total hysterectomy has been proven to present many advantages over the other approaches. Therefore, it seems that this route should also be applied in the case of subtotal hysterectomy. We present 9 cases of patients who underwent subtotal hysterectomy performed through the vagina for benign gynecological diseases.
\end{abstract}

Key words: hysterectomy, vaginal gynecological surgical procedures, uterine diseases.

\section{Introduction}

Hysterectomy is one of the most common procedures in gynecologic surgery for benign diseases. There are three main approaches to this intervention: abdominal, vaginal and laparoscopic [1]. The procedure can be performed in two ways: by removing the whole uterus including the cervix (total hysterectomy $-\mathrm{TH}$ ) or excising only the body of the uterus and leaving the cervix intact (subtotal, also known as supracervical hysterectomy - STH) [2]. The first operation of total transabdominal hysterectomy was successfully performed in 1929 [3]. It was before the introduction of regular screening for cervical carcinoma; therefore, removal of the potentially hazardous transformation zone was essential. Since Pap smear was brought into practice, STH has become an acceptable form of surgery. Nevertheless, this procedure is not very popular among surgeons. A recent questionnaire, which was carried out in the United States among 770 gynecologists, showed that only $19 \%$ of the respondents offered a choice between $\mathrm{TH}$ and STH to their patients [4]. Removing the cervix at hysterectomy has been a controversial issue over the past few years. Some authors believe that abdominal STH is an intervention suited mainly to inexperienced gynecologists as it is easy to learn and does not require risky dissection in the proximity of the ureters [5]. For about 30 years now surgeons have discussed the potential predominance of STH over TH with regard to sexual, urinary and bowel function and pelvic organ prolapse. However, it seems that the most important factor to be taken into consideration when deciding whether the cervix should be removed is patient preference [2].

The route of hysterectomy for benign diseases was also evaluated in the recent Cochrane Review of 34 randomized trials [6]. It was concluded that the vaginal procedure should be preferably chosen by surgeons as it has the best outcome in comparison with both abdominal and laparoscopic approaches.

Subtotal hysterectomy is not usually performed vaginally; however, in the light of the circumstances mentioned above, this route seems to be an interesting alternative to the traditional approaches. 


\section{Aim}

Some patients desire to leave their cervix intact. In those cases supracervical hysterectomy is necessary. The vaginal route in gynecological surgery is less invasive and has many advantages over traditional approaches. We decided to present cases of supracervical hysterectomy performed vaginally. It is a very rare technique in gynecological surgery for benign diseases.

\section{Material and methods}

Subtotal hysterectomy was performed in 9 patients who were admitted to our ward between 2003 and 2012 (Table I). All patients suffered from benign gynecologic diseases that were confirmed by clinical and ultrasound evaluations.

Refractory benign gynecological diseases are a common reason for a hysterectomy procedure [7]. Despite the fact that conservative treatment was introduced before the surgery, patients still suffered from persistent uterine bleeding and painful menstruation. Their symptoms were troublesome and all patients decided to choose the surgical way of treatment. Hormonal therapy (progestins) and iron supplementation were prescribed to improve their clinical condition before the operation. Thanks to that haemoglobin (HGB) levels were normal. The selection of patients is based, among other things, on the uterine body volume since it is easier to perform that kind of procedure when the uterus is not too large. This is the reason why the uterine body volume in the group of our patients was low. A large uterus should be considered as a contraindication to the supracervical vaginal hysterectomy.

It should be emphasized that all 9 patients strongly desired to leave their cervix intact and did not accept the idea of performing total hysterectomy.

\section{Technique of subtotal hysterectomy}

The patient is placed in a lithotomy position and bladder catheterization is performed. Two tenacula positioned on the anterior part of the cervix allow for manipulation of the uterus during the operation. Then, the vaginal part of the cervix is injected with a solution of $0.9 \% \mathrm{NaCl}$ and adrenaline (1: 200 000) in order to reduce the bleeding. Anterior colpotomy and posterior colpotomy are performed. The bladder is dissected and moved upwards to protect it from any injury (Photos 1 and 2). After opening of the anterior and posterior peritoneal folds, the uterus can be easily manipulated and access to all anatomical structures is possible. The uterine body is delivered into the vagina through the anterior colpotomy and the cervix is pushed inside the peritoneal cavity through the posterior colpotomy (Photo 3). If this is not possible, morcellation or debulking may be used in order to reduce the volume of the uterus. Round ligaments, proper ovarian ligaments, Fallopian tubes and uterine vessels can be easily visualized, secured and cut (Photos 4 and 5). Once it is done, supracervical amputation of the uterus is performed (Photo 6). At this point, a reversed conisation or electrocoagulation of the endometrial tissue localized in the endocervix could be considered as potentially beneficial to the patients [8]. Closure of the wound is performed by continuous suture and the cervix is placed in its normal position (Photo 7). Incisions made during anterior and posterior colpotomy are closed by continuous sutures and a vaginal pack is inserted.

All 9 operations were performed by the third author of this article.

\section{Results}

The blood loss and the duration of the surgery were acceptable and did not differ from other vaginal surgeries usually performed in our ward. Apart from one patient reporting a persistent headache and emesis for the duration of 2 days, the postoperative period was uneventful. The length of a medium postoperative hospital stay was similar to the other vaginal operations performed in our ward and lasted approximately 3 days. Basic information concerning patient characteristics and details of the intra- and postoperative period are presented in the Table I.

\section{Discussion}

A large meta-analysis of 34 studies that included 4495 women, concerning benefits and disadvantages of abdominal, vaginal and laparoscopic hysterectomy, was published in 2009 by Nieboer et al. [6]. Their conclusion was that vaginal hysterectomy was significantly better than the abdominal procedure and should be chosen preferably, when possible. Results of the meta-analysis showed that vaginal hysterectomy compared to the abdominal surgery has many advantages in terms of earlier return to nor- 


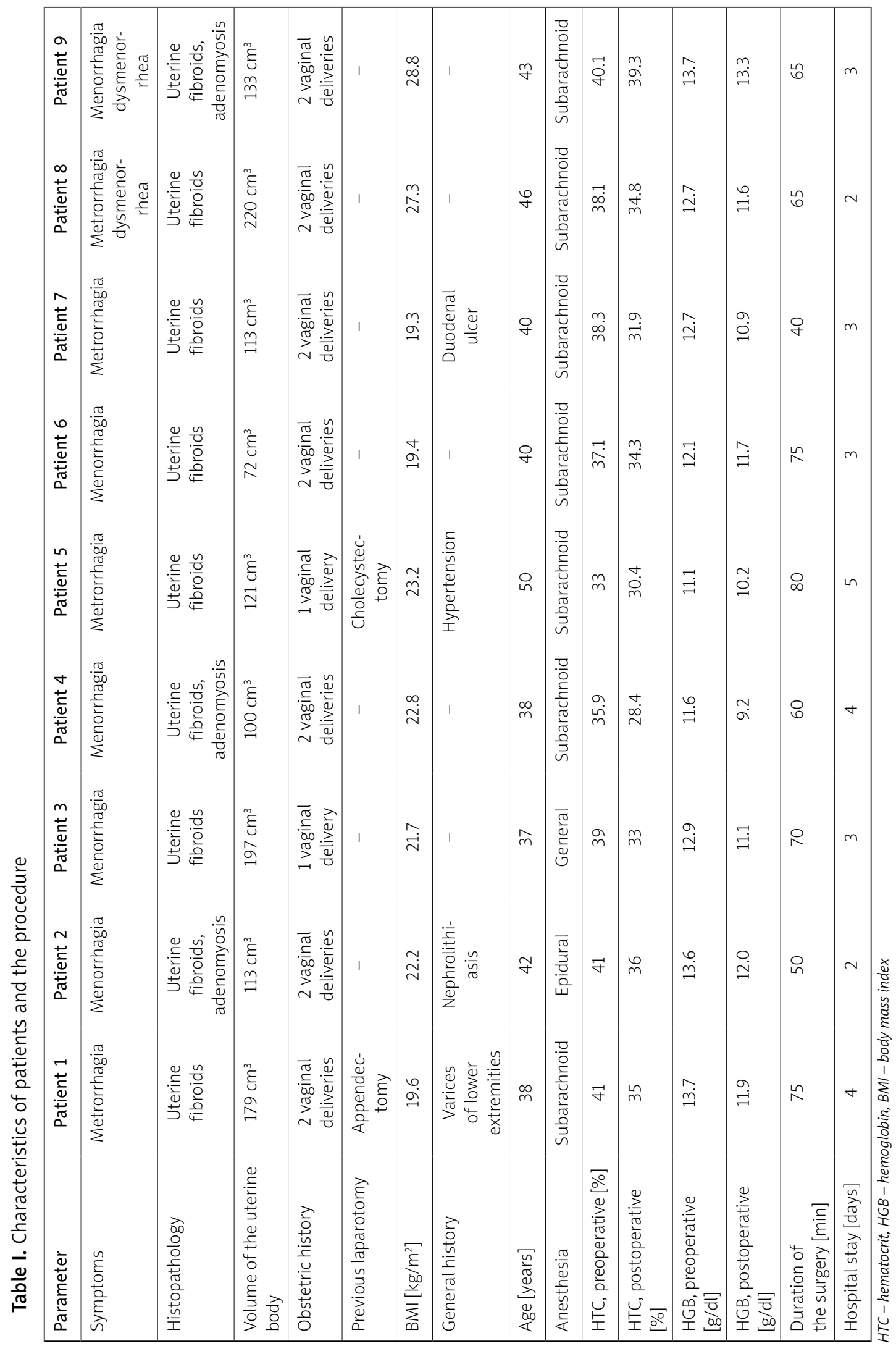




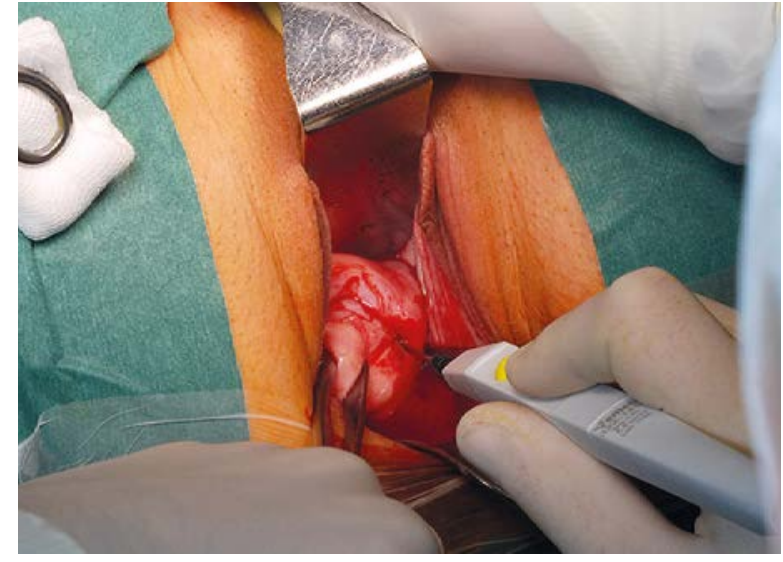

Photo 1. Performing anterior colpotomy

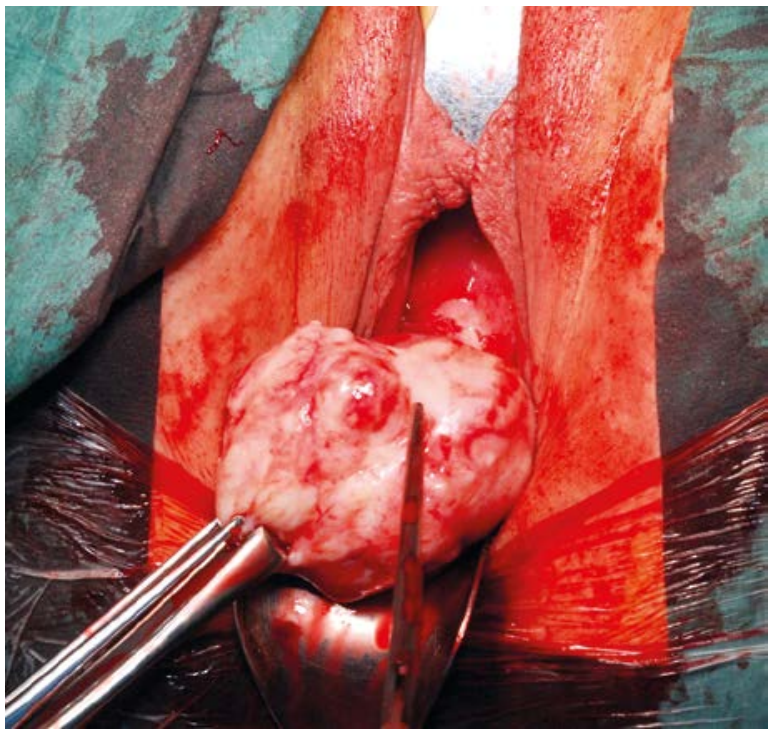

Photo 3. Delivering the uterine body with myoma from the peritoneal cavity

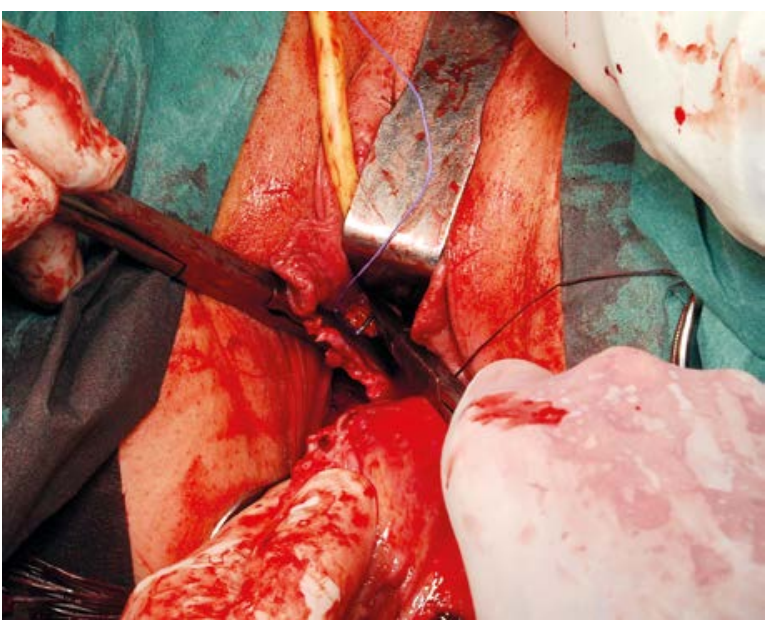

Photo 5. The right uterine pedicles being clamped and ligated

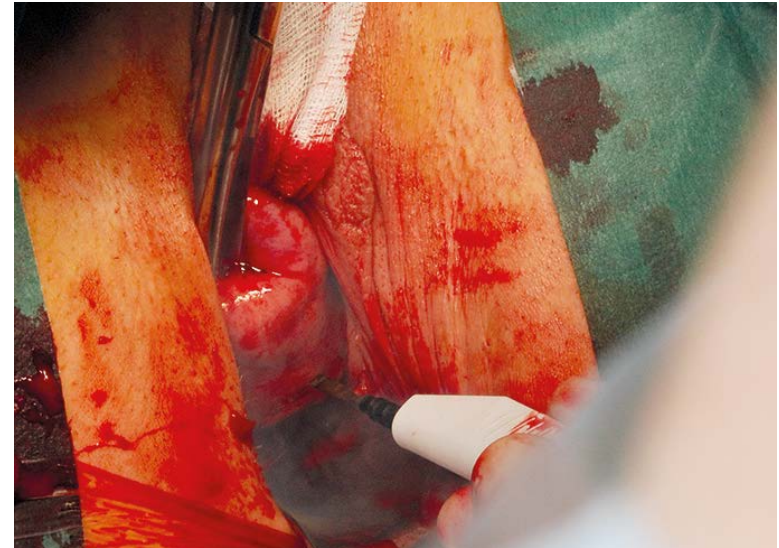

Photo 2. Performing posterior colpotomy

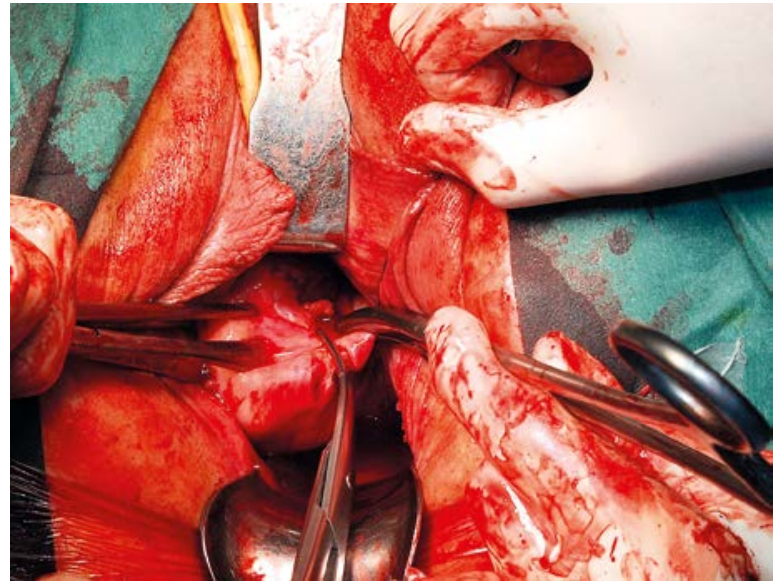

Photo 4. The left ovarian pedicles being clamped and cut

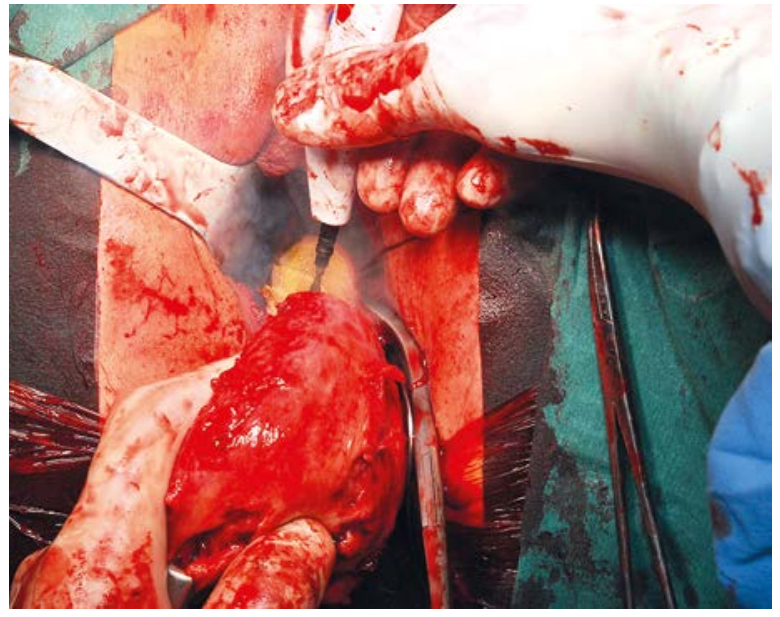

Photo 6. The body of the uterus being amputated from the cervix 
mal activities and discharge from the hospital, fewer febrile episodes and quicker recovery associated with less postoperative pain. Laparoscopy should be considered only when a vaginal route is contraindicated as it requires longer operating time and causes greater postoperative pain and higher costs [6]. However, there are some contraindications to vaginal hysterectomy that have to be discussed before the operation, namely no prior vaginal delivery, previous laparotomy, limited vaginal access, large (greater than 12/14-week pregnancy) or immobile uterus, suspicion of genital malignancy and previous vesicovaginal fistula repair [9-12]. On the other hand, some authors believe that it is possible to perform the operation safely even if these contraindications are present [13]. Debulking or morcellation of the uterus could be useful in some of these situations. After taking all these facts into consideration, it seems that the vaginal route could be applied in the majority of benign gynecologic diseases. However, although, clearly, vaginal STH might have some advantages over abdominal or laparoscopic procedures, it should be emphasized that the procedure is limited by all contraindications mentioned above. Appropriate mobility of the cervix, good vaginal access and the size of the uterus smaller than consistent with 12 weeks of pregnancy, as well as previous vaginal deliveries, seem to be essential to perform this procedure. We strongly advise not to perform vaginal STH when these conditions are not fulfilled. The technique of the surgery requires enough space in the operating field to deliver the uterus through the anterior colpotomy. The use of GnRH analogues before the operation could be an alternative if the uterus is too large. Selection of candidates for the surgery has to be carried out much more carefully than in the case of traditional total vaginal hysterectomy.

Due to the fact that the procedure of vaginal STH is not very popular among surgeons, published data are scarce. Nevertheless, an interesting review by Thomas and Magos was published in 2011 [2]. The authors described briefly the surgical technique used in their hospital, concluding that the vaginal route for STH is easy to learn and definitely worth wider application. In comparison to our technique, they performed only anterior semi-circular colpotomy. Using clamps or sutures, a bite was taken into the uterus. Simultaneous downward traction and taking other bites as high as possible allowed the uterus to be delivered into the vagina [2].

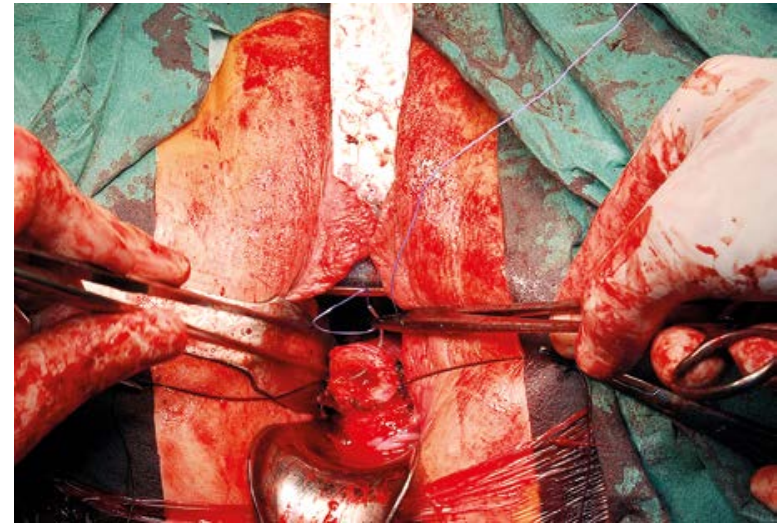

Photo 7. Suturing the cervical stump

Over the past few years many controversies have arisen concerning disadvantages and advantages of $\mathrm{STH}$ in comparison to TH. It was suggested that regional innervation and disruption of ligaments during $\mathrm{TH}$ might negatively influence pelvic organ functions and patient sexuality after the surgery. In the 1980s, Kilkku et al. stated that preserving the cervix may lead to some sexual benefits [14]. However, more recent studies showed that the improvement of sexuality is equal in patients who underwent TH or STH. The reduction of dyspareunia is obtained in both cases and there is no evidence for the superiority of one procedure over the other [15]. The latest Cochrane Review, which collected evidence based on three randomized controlled trials, showed that there is no significant difference between these two methods in terms of sexual satisfaction, constipation and urinary incontinence after the surgery [16]. A similar conclusion was drawn by Thakar et al. [17]. Bowel and bladder function do not differ postoperatively after 12 months in women who underwent TH compared to STH [18].

Intraoperative and postoperative outcomes were also evaluated in the Cochrane Review. The authors concluded that there is a benefit of STH in terms of operating time and blood loss. The risk of intra-abdominal hematoma, as well as the duration of the hospital stay, were factors that favored STH; however, that difference was not statistically significant [18]. A randomized trial by Thakar et al. indicated that the rate of pyrexia in the group of women who underwent TH was higher (6\% vs. 19\%) [18].

The pelvic prolapse rate was evaluated in the Cochrane Review and showed no difference between STH and TH procedures. However, some authors found that the total procedure provided better outcomes. Virtanen et al. reported that $6.2 \%$ of women 
after STH had a prolapse, in contrast to $2.2 \%$ of patients undergoing TH [19].

Menstruation can be more likely after the STH procedure, which is associated with residual endometrial tissue left in the cervix. The patient should be informed that in some cases it is not possible to obtain amenorrhea after the procedure. Gynecologists ought to remember that hormonal therapy should consist of estrogen opposed with progestogen to reduce the risk of endometrial cancer [2].

Leaving the cervix intact is not recommended for women with previously abnormal cervical smears. The risk of development of cervical stump carcinoma in women with no prior abnormality is about $0.3 \%$ [20]. Introduction of effective screening allowed surgeons to perform STH; however, patients should be counseled about the necessity of continuing regular Pap smear control [21].

\section{Conclusions}

It is important to remember that the decision whether to remove or retain the cervix belongs to the patient. The surgeon should inform the woman about all advantages and disadvantages that are discussed above [2]. Benefits of the vaginal route and STH can be combined together, creating another option of a surgical procedure that could meet the needs of patients and gynecologists.

\section{References}

1. Garry R, Fountain J, Mason S, et al. The evaluate study: two parallel randomized trials, one comparing laparoscopic with abdominal hysterectomy, the other comparing laparoscopic with vaginal hysterectomy. BMJ 2004; 328: 129-33.

2. Thomas B, Magos A. Subtotal hysterectomy and myomectomy - vaginally. Best Pract Res Clin Obstet Gynaecol 2011; 25: 133-52.

3. Richardson EH. A simplified technique for abdominal panhysterectomy. Surg Gynecol Obstet 1929; 48: 248-51.

4. Zekam N, Oyelese Y, Goodwin K, et al. Total versus subtotal hysterectomy: a survey of gynecologists. Obstet Gynecol 2003; 102: 301-5.

5. Parys BT, Haylen BT, Hutton JL, et al. Urodynamic evaluation of lower urinary tract function in relation to total hysterectomy. Aust N Z J Obstet Gynecol 1990; 30: 161-5.

6. Nieboer TE, Johnson N, Lethaby A, et al. Surgical approach to hysterectomy for benign gynecological disease. Cochrane Database Syst Rev 2009; 8: CD003677.

7. Kramer MG, Reiter RC. Hysterectomy: indications, alternatives and predictors. Am Fam Physician 1997; 55: 827-34.

8. Rokita W, Stanisławska M, Spaczyński M, et al. Electrosurgery of cervical changes and its place in cervical cancer prophylaxis. Ginekol Pol 2009; 80: 856-60.
9. Kluivers KB, Hendriks JCM, Mol BWJ, et al. Quality of life and surgical outcome after total laparoscopic hysterectomy versus total abdominal hysterectomy for benign disease: a randomized, controlled trial. J Minim Invasive Gynecol 2007; 14: 145-52.

10. Ferrari MM, Berlanda N, Mezzopane R, et al. Identifying the indications for laparoscopically assisted vaginal hysterectomy: a prospective, randomized comparison with abdominal hysterectomy in patients with symptomatic uterine fibroids. BJOG 2000; 107: 620-5.

11. Seracchioli R, Venturoli S, Vianello F, et al. Total laparoscopic hysterectomy compared with abdominal hysterectomy in the presence of a large uterus. J Am Assoc Gynecol Laparosc 2002; 9: 333-8.

12. Dorsey JH, Steinberg EP, Holtz PM. Clinical indications for hysterectomy route: patient characteristics or physician preference? Am J Obstet Gynecol 1995; 173: 1452-60.

13. Doucette RC, Sharp HT, Alder SC. Challenging generally accepted contraindications to vaginal hysterectomy. Am J Obstet Gynecol 2001; 184: 1386-9.

14. Kilkku P, Gronroos M, Hirvonen T, et al. Supravaginal uterine amputation vs. hysterectomy. Effects on libido and orgasm. Acta Obstet Gynecol Scand 1982; 61: 265-7.

15. Zobbe V, Gimbel H, Andersen BM, et al. Sexuality after total vs. subtotal hysterectomy. Acta Obstet Gynecol Scand 2004; 83: 191-6.

16. Lethaby A, Ivanova V, Johnson N. Total versus subtotal hysterectomy for benign gynaecological conditions. Cochrane Database Syst Rev 2006; 19: CD004993.

17. Thakar R, Ayers S, Srivastava R, et al. Removing the cervix at hysterectomy: an unnecessary intervention? Obstet Gynecol 2008; 112: 1262-9.

18. Thakar R, Ayers S, Clarkson P, et al. Outcomes after total versus subtotal abdominal hysterectomy. N Engl I Med 2002; 347: 1318-25.

19. Virtanen H, Makinen J, Teho T, et al. Effects of abdominal hysterectomy on urinary and sexual symptoms. Br J Urol 1993; 72: 868-72.

20. Storm HH, Clemmensen IH, Manders T, et al. Supravaginal uterine amputation in Denmark 1978-1988 and risk of cancer. Gynecol Oncol 1992; 45: 198-201.

21. Grabiec M, Walentowicz M, Marszatek A. Multiple skin metastases to vulva from carcinoma of the cervical stump. Ginekol Pol 2010; 81: 140-3.

Received: 22.07.2013, accepted: 11.10.2013 